Diclofenac for intermediate-risk PE

Thromb Res

\title{
Diclofenac for reversal of right ventricular dysfunction in acute normotensive pulmonary embolism: a pilot study
}

\begin{abstract}
Authors:
David Jimenez ${ }^{1}$, Rosa Nieto ${ }^{1}$, Jesús Corres ${ }^{2}$, Covadonga Fernández-Golfín ${ }^{3}$, Deisy Barrios ${ }^{1}$, Raquel Morillo ${ }^{1}$, Carlos Andres Quezada ${ }^{1}$, Menno Huisman ${ }^{4}$, Roger D. Yusen ${ }^{5}$, Jeffrey Kline ${ }^{6}$
\end{abstract}

\begin{abstract}
Affiliation:
${ }^{1}$ Respiratory Department, Hospital Ramón y Cajal and Medicine Department, Universidad de Alcalá (IRYCIS), Madrid, Spain.

${ }^{2}$ Emergency Department, Hospital Ramón y Cajal, Madrid, Spain.

${ }^{3}$ Cardiology Department, Hospital Ramón y Cajal and Medicine Department, Universidad de Alcalá (IRYCIS), Madrid, Spain.

${ }^{4}$ Department of Thrombosis and Hemostasis, Leiden University Medical Center, Leiden, the Netherlands

${ }^{5}$ Divisions of Pulmonary and Critical Care Medicine and General Medical Sciences, Washington University School of Medicine, St. Louis, Missouri, USA.

${ }^{6}$ Department of Emergency Medicine, Indiana University School of Medicine, Indianapolis, IN, USA.
\end{abstract}


Diclofenac for intermediate-risk PE

Thromb Res

\section{Correspondence:}

David Jiménez

Respiratory Department, Hospital Ramón y Cajal and Medicine Department, Universidad de Alcalá (IRYCIS), 28034 Madrid, Spain

Phone: +34913368314

e-mail: djimenez.hrc@gmail.com

Short title: Diclofenac for intermediate-risk PE

Tables: 3

Figures: 3

Word count: 3,399

Funding: Ministerio de Economía, Industria y Competitividad (PI11/00246) 
Diclofenac for intermediate-risk PE Thromb Res

\section{ABBREVIATION LIST}

BNP: brain natriuretic peptide

Cl: confidence interval

cox: cyclooxygenase

CT: computed tomography

HFABP: heart-type fatty acid-binding protein

IVC: inferior vena cava

LMWH: low-molecular weigh heparin

NSAIDs: nonsteroidal anti-inflammatory drugs

PE: pulmonary embolism

RV: right ventricle

SD: standard deviation

TIPES: Tenecteplase Italian Pulmonary Embolism Study

UFH: unfractionated heparin

V/Q: ventilation-perfusion 
Diclofenac for intermediate-risk PE

Thromb Res

\section{ABSTRACT}

Background: The inflammatory response associated with acute pulmonary embolism (PE) contributes to the development of right ventricular (RV) dysfunction. Nonsteroidal anti-inflammatory drugs (NSAIDs) may facilitate the reversal of PE-associated RV dysfunction.

Methods: We randomly assigned normotensive patients who had acute PE associated with echocardiographic RV dysfunction and normal systemic blood pressure to receive intravenous (IV) diclofenac (two doses of $75 \mathrm{mg}$ in the first 24 hours after diagnosis) or IV placebo. All patients received standard anticoagulation with subcutaneous low-molecular-weight heparin (LMWH) and an oral vitamin $\mathrm{K}$ antagonist. $\mathrm{RV}$ dysfunction was defined by the presence of, at least, two of the following criteria: i) RV diastolic diameter $>30 \mathrm{~mm}$ in the parasternal window; ii) RV diameter > left ventricle diameter in the apical or subcostal space; iii) RV free wall hypokinesis; and iv) estimated pulmonary artery systolic pressure $>30 \mathrm{~mm} \mathrm{Hg}$. Persistence of RV dysfunction at 48 hours and 7 days after randomization were the primary and secondary efficacy outcomes, respectively. The primary safety outcome was major bleeding within 7 days after randomization.

Results: Of the 34 patients randomly assigned to diclofenac (17) or placebo (17), the intention-to-treat analysis showed persistent RV dysfunction at 48 hours in $59 \%$ (95\% confidence interval [Cl], 33-82\%) of the diclofenac group and in $76 \%$ $(95 \% \mathrm{Cl}, 50-93 \%)$ of the placebo group (difference in risk [diclofenac minus standard anticoagulation], -17 percentage points; $95 \% \mathrm{Cl},-47$ to 17 ). Similar proportions $(35 \%)$ of patients in the diclofenac and placebo groups had persistent $\mathrm{RV}$ dysfunction at 7 days. Major bleeding occurred in none of patients in the diclofenac group and in $5.9 \%(95 \% \mathrm{Cl}, 0.2-29 \%)$ of patient in the placebo group. 
Diclofenac for intermediate-risk PE

Thromb Res

Conclusions: Due to slow recruitment, our study is inconclusive as to a potential benefit of diclofenac over placebo to reverse RV dysfunction in normotensive patients with acute PE.

Clinical Trial Registration: URL: http://www.clinicaltrials.gov. Unique identifier: NCT01590342. 
Diclofenac for intermediate-risk PE

Thromb Res

\section{INTRODUCTION}

In patients with acute pulmonary embolism (PE), right ventricular (RV) dysfunction results from mechanical obstruction of the pulmonary vasculature, pulmonary arterial vasoconstriction, and inflammation of the RV muscle (1). Hypothesized mechanisms of RV inflammation include the accumulation of neutrophils and monocytes in the RV outflow track, and elevated circulating concentrations of myeloperoxidase and other inflammatory biomarkers associated with PE $(2,3)$.

Parenteral anticoagulation remains the primary acute treatment for PE (4). Heparins inhibit the action of thrombin, allowing the unopposed effect of endogenous plasmin to degrade clot volume over a period of days to weeks (5). Since the acute development of RV dysfunction in association with acute PE increases the risk of death from PE, studies have assessed the efficacy and safety of thrombolytic therapy for these patients (6). Fibrinolytic agents allow faster clot size reduction than heparins, but they have an increased risk of haemorrhage (7). In addition, approximately $16 \%$ of patients with PE have a contraindication to anticoagulation, and $30 \%$ have a contraindication to fibrinolysis (8).

Attenuation of the inflammatory response in patients who have acute PE and associated with RV dysfunction may improve patient outcomes. A rat model of PE by Watts et al suggested that administration of ketorolac reduced RV inflammatory genes, reduced neutrophil influx, and improved RV function in the setting of experimental PE (9). This study assessed the efficacy of diclofenac for improving $\mathrm{RV}$ dysfunction in intermediate-risk PE patients who received standard anticoagulation.

\section{METHODS}




\section{Study design}

The AINEP trial (NCT01590342) was a single center prospective, randomized, blinded, parallel-group, placebo-controlled study that evaluated the hypothesis that therapy with diclofenac would improve RV function in patients who had intermediate-risk acute PE and associated RV dysfunction. Patients were recruited between 2013 and 2016 from a tertiary university hospital in Madrid, Spain. The study was conducted in accordance with the Declaration of Helsinki, was approved by the local ethics committee, and required written informed consent for participation.

\section{Study patients}

Consecutive adult patients who had an objective diagnosis of PE were considered for inclusion in the study if they had: 1) onset of symptoms since no more than 10 days, 2) normal blood pressure (systolic blood pressure $>100$ $\mathrm{mmHg}$ ), and 3) echocardiographic RV dysfunction detected within 12 hours after the diagnosis of $\mathrm{PE}$. For the diagnosis of PE, confirmatory testing required a high probability ventilation-perfusion (V/Q) scintigraphy (10), or a positive contrastenhanced, PE-protocol, helical chest multi-detector computerized tomography (CT) for PE (11).

Study exclusion criteria consisted of a history of chronic pulmonary hypertension, bronchial asthma, or severe heart failure. Patients were also excluded if they had had clinically relevant bleeding, major surgery or trauma within the last month, or if they had a hemorrhagic diathesis, active peptic ulcer, Crohn's disease or ulcerative colitis, or severe hepatic or renal failure. Exclusion criteria also included use of oral anticoagulation in the last two weeks, pregnancy, lactation, or less than 30 days post-partum.

\section{Randomization and treatment}


Diclofenac for intermediate-risk PE

Thromb Res

Eligible patients were randomly allocated in a 1:1 ratio to receive diclofenac or placebo. Treatments were randomly allocated at the Pharmacy of Ramon y Cajal Hospital (Madrid, Spain) with a computer-generated list of random number in blocks of four. Copies of the randomization code were kept in sealed, sequentially numbered, opaque envelopes at the pharmacy.

Patients who were assigned to receive diclofenac were given an intravenous (IV) infusion of diclofenac $(75 \mathrm{mg}, 3 \mathrm{~mL})$ given over 30 minutes, two doses 12 hours apart in the first 24 hours after randomization. Patients assigned to placebo were given an IV infusion that had the same volume and appearance as for diclofenac, and they had a similar infusion duration and schedule. Diclofenac and placebo solutions were provided by the local pharmacy.

All patients received standard anticoagulation with subcutaneous low-molecularweight heparin (LMWH) enoxaparin (1 $\mathrm{mg} / \mathrm{Kg} / 12$ hours). An oral vitamin $\mathrm{K}$ antagonist was started on the third day after randomization. LWMH was discontinued after the INR was at least 2.0 for two consecutive days.

\section{Echocardiography assessment}

The study required that patients undergo TTE within 12 hours after diagnosis of PE. Trained and certified local cardiologists, blinded to the patient's clinical data and the time of the echocardiography (i.e., before or after treatment), interpreted each echocardiogram, and these measurements were used for analyses. The study defined echocardiographic RV dysfunction as the presence of, at least, two of the following criteria: i) RV diastolic diameter $>30 \mathrm{~mm}$ in the parasternal window; ii) RV diameter > left ventricle diameter in the apical or subcostal space; iii) RV free wall hypokinesis; and iv) estimated pulmonary artery systolic pressure $>30 \mathrm{~mm} \mathrm{Hg}(12)$. 
Diclofenac for intermediate-risk PE

Thromb Res

A core laboratory that was blinded to group assignment measured baseline $\mathrm{RV} / \mathrm{LV}$ ratios, and these measures were compared with the data obtained by local cardiologists to assess interobserver agreement.

\section{Follow-up and outcome assessment}

Study personnel assessed study participants for death, hemodynamic decompensation (or collapse), bleeding, stroke, recurrent PE, and serious adverse events for 30 days post-randomization. An independent clinical-events committee, whose members were unaware of the treatment-group assignments, adjudicated all efficacy and safety outcomes.

The primary efficacy outcome of the study was the resolution of echocardiographic RV dysfunction at 48 hours after randomization. The secondary efficacy outcome was the resolution of RV dysfunction at 7 days after randomization. The primary safety outcome was major bleeding within 7 days after randomization. Major bleeding was defined as overt bleeding associated with a fall in the hemoglobin level of at least $2.0 \mathrm{~g} / \mathrm{dl}$ or with transfusion of $\geq 2$ units of red blood cells, with involvement of a critical site (intracranial, intraspinal, intraocular, retroperitoneal, intraarticular or pericardial, or intramuscular with compartment syndrome), or with death (13).

\section{Statistical analyses}

Sample size assumptions were based on the Tenecteplase Italian Pulmonary Embolism Study (TIPES), in which therapy with weight-adjusted intravenous tenecteplase significantly reduced the $R V$ / left ventricle (LV) ratio at 24 hours by a mean of 0.31 (standard deviation [SD] 0.20 ), and therapy with unfractionated heparin (UFH) alone resulted in a nonsignificant reduction in $\mathrm{RV} / \mathrm{LV}$ ratio by a mean of 0.10 (SD 0.30) (14). We assumed that diclofenac would have an effect similar to tenecteplase for resolution of RV dysfunction, and we used the means and SDs of the difference in RV/LV ratio from the tenecteplase and heparin groups for sample size calculation. Using a beta of 0.8 
Diclofenac for intermediate-risk PE

Thromb Res

(power of $80 \%$ ), and a 2 -sided alpha of 0.05 , the estimated sample size was 45 per group. Because of slow recruitment and limited resources, the trial closed in December 2016.

Continuous data are presented as mean $\pm S D$ or, in case of a skewed distribution, as median values with ranges. Comparison of binary data between the groups was performed with the Fisher exact test. Within-group comparisons of continuous data were performed with the 2 -sided paired $t$ test. Within-group ordinal data were compared with the 2-sided Wilcoxon signed-rank test. Between-group continuous data were compared with the 2-sided unpaired t test or Wilcoxon rank sum test. Between-group ordinal data were compared with the exact Mantel- Haenszel chi-square test. Interobserver agreement for the echocardiographic baseline $R V / L V$ ratio between the investigator and core laboratory measurements was assessed by Bland-Altman analysis (15).

\section{RESULTS}

\section{Patients}

From January 2013 through December 2016, a total of 34 patients were enrolled. Of these patients, 17 were randomly assigned to treatment with diclofenac, and 17 were randomly assigned to placebo (Figure 1). The two treatment groups had similar baseline demographic, clinical, and medical history data (Table 1). The median age in the study population was 75 years and $65 \%$ were women. All patients were normotensive at randomization.

\section{Echocardiography}

With all patients having $\mathrm{RV}$ dysfunction at randomization, it persisted in $59 \%$ (95\% confidence interval [CI], 33-82\%) of the diclofenac group and in $76 \%(95 \%$ $\mathrm{Cl}, 50-93 \%$ ) of the placebo group at 48 hours (Table 2), which did not meet statistical significance for the primary efficacy outcome (59\% vs. $76 \%$, 
respectively; $P=0.46$ ). By day 7 , the diclofenac and placebo groups had similar proportions (35\%) of patients who had persistent RV dysfunction (Table 2).

The two treatment groups had similar echocardiographic mean RV end-diastolic diameter $(45 \pm 9.8 \mathrm{~mm}$ and $40 \pm 6.3 \mathrm{~mm}$ in the diclofenac and placebo groups, respectively; $P=0.15)$ and $R V / L V$ ratio $(1.2 \pm 0.2$ and $1.3 \pm 0.02$ in the diclofenac and placebo groups, respectively; $P=0.31$ ) at baseline. At 48 hours postrandomization, the diclofenac and placebo groups did not show a significant difference in within-group mean change score for $\mathrm{RV}$ end-diastolic dimension $(0.6 \pm 0.7$ [diclofenac] vs. $0.3 \pm 0.7$ [placebo]; $P=0.26)$, or for $R V / L V$ ratio $(0.2 \pm$ 0.2 vs. $0.2 \pm 0.3 ; P=0.67$ ) (Figure 2). Similarly, at 7 days post randomization, the diclofenac and placebo groups did not show a significant difference in withingroup mean change score for $R V$ end-diastolic dimension (1.1 \pm 1.1 [diclofenac] vs. $0.9 \pm 0.5$ [placebo]; $P=0.45)$ or for $R V / L V$ ratio $(0.3 \pm 0.3$ vs. $0.4 \pm 0.3 ; P=$ $0.73)$.

\section{Safety outcomes}

Only one major bleeding event occurred (Table 3). An 81 year-old patient in the placebo group developed cardiovascular collapse 3 days after initially being treated with anticoagulant therapy. He received "rescue" thrombolytic therapy. Two days later, a major gastrointestinal bleeding event led to discontinuation of anticoagulation and placement of an inferior vena cava (IVC) filter. Sixteen days after the diagnosis of acute PE, he developed a new symptomatic deep vein thrombosis. At 30 days, none of the 34 randomized patients had died.

\section{Interobserver agreement}

In 32 (94\%; 95\% Cl, 80-99\%) of 34 analyzed patients, there was agreement between investigators and core laboratory for identifying patients with a baseline $R V / L V$ ratio $>1$. The mean $( \pm S D)$ difference in the baseline echocardiographic $\mathrm{RV} / \mathrm{LV}$ ratio between investigator and core laboratory measurements was $0.03 \pm$ 0.10 (Figure 3). 


\section{DISCUSSION}

We conducted the first registered (clinicaltrials.gov) randomized trial of diclofenac versus placebo in normotensive patients who had acute symptomatic PE associated with RV dysfunction. Between the two randomized treatment groups, this study did not detect a statistically significantly greater improvement in the proportion of patients who had $\mathrm{RV}$ dysfunction at 48 hours or at 7 days after randomization. Many patients in both randomized groups had improvements in multiple echocardiographic variables related to RV dysfunction at 48 hours, and most patients showed such improvement at 7 days after randomization, and statistically significant within group improvements occurred for most variables at both time points. Since poor enrollment led to early termination of the study, a type II error could have occurred, since the study only had a power of $18 \%$ to detect a difference in the primary outcome. By extrapolation, these data imply that a follow-up study in a similar patient population would require 118 randomized patients in each treatment group to be able to detect a betweengroup difference of $17 \%$ in the proportion of patients who normalized their echocardiographic findings. Thus, this work provides necessary preliminary data for a larger and more definitive clinical trial.

This trial emphasizes an important ancillary point about the difficulty of executing an acute treatment trial for patients with PE and RV dysfunction. Despite 15 consecutive hours per day of pre-screening and screening for eligible subjects over a five-year period in a large, tertiary treatment center, only 88 of 213 patients approached were eligible (41\%), and only 34 (16\% of patients approached and $39 \%$ of those eligible) agreed to participate.

Supporting the rationale for this study and the non-statistically significant results demonstrated in this study, a rat model of PE by Watts et al demonstrated that 
Diclofenac for intermediate-risk PE

Thromb Res

administration of ketorolac protected RV contractile function in the setting of experimental PE (9). Regarding our choice of NSAID, we decided to use diclofenac, a nonselective cyclooxygenase (COX)-1 and COX-2 inhibitor (19), since COX-2 inhibitors may increase the risk of platelet activation and vasoconstriction (16), and studies have demonstrated their association with increased mortality in chronic heart failure (17) and myocardial infarction (18). We chose to use a dose of diclofenac that studies of analgesia successfully used $(20,21)$.

Regarding the safety of NSAID use among patients with venous thromboembolism receiving anticoagulant therapy, studies have shown an association between NSAID use and an increased risk of clinically relevant and major bleeding (22). Although we did not design this trial of diclofenac vs placebo to meet non-inferiority criteria for bleeding complications, we so far feel reassured that none of the patients randomized to the 2 doses of diclofenac had a major bleeding complication. Of note, the eligibility criteria for the study did exclude some of the patients at higher risk for bleeding.

Different groups of investigators have not agreed upon standard definitions for echocardiographic findings of RV dysfunction (23). However, previous studies supported the use of this study's primary outcome (24). Similar to a previous trial that assessed the efficacy and safety of catheter-directed thrombolysis in patients who enrolled with intermediate-risk $\mathrm{PE}$, our data suggested an excellent core lab / local clinician agreement of $94 \%$ for identifying patients a baseline $\mathrm{RV} / \mathrm{LV}$ ratio $>1$. Also supporting the use of $R V$ dysfunction for assessing efficacy, our study showed that resolution of RV dysfunction was achieved in $65 \%$ of patients at 7 days in both randomized groups, similar to many randomized clinical trials of rt-PA versus heparin that demonstrated a catch-up phenomenon in the heparin group, with initially worse hemodynamic parameters that later became similar to the thrombolytic group at 1 week of follow-up and beyond (25). Futures studies could assess for NSAID effects on other 
Diclofenac for intermediate-risk PE

Thromb Res

echocardiographic measurements that we did not assess (26). A larger study could also have greater power to look for more clinically relevant outcomes such as survival.

The most obvious limitations of this study include the inability to successfully enroll an adequate number patient in a timely manner, and the subsequently reduced power to detect differences between treatment groups. Even if future studies confirm the efficacy of diclofenac, the most efficacious and safe drug dose and schedule remains unclear. Unfortunately, we did not systematically collect cardiac biomarkers assessing myocardial injury (i.e., troponin or hearttype fatty acid-binding protein [HFABP])) or RV stress (i.e., brain natriuretic peptide [BNP]), which could have helped us understand and interpret the results of the trial (27). We do not believe that the results of this trial are a consequence of inappropriate patient selection. From a biological point of view, the relative effect of the intervention (i.e., diclofenac) on the outcome should be the same across the spectrum of PE severity, whereas absolute risk reductions will depend on the baseline risk of experiencing the outcome. Thus, it seems reasonable to support future trials of NSAIDS in patients who have normotensive acute PE associated with $\mathrm{RV}$ dysfunction and intermediate-risk for death. 
Diclofenac for intermediate-risk PE Thromb Res

\section{Declaration of interests}

None to be disclosed. 
Diclofenac for intermediate-risk PE

Thromb Res

\section{Author contributions}

Study concept and design: Jimenez, Yusen, Kline.

Acquisition of data; analysis and interpretation of data; statistical analysis:

Jimenez, Nieto, Corres, Fernández-Golfín, Barrios, Morillo, Quezada, Huisman, Yusen, Kline.

Drafting of the manuscript: Jimenez, Huisman, Yusen, Kline.

Critical revision of the manuscript for important intellectual content: Jimenez, Nieto, Corres, Fernández-Golfín, Barrios, Morillo, Quezada, Huisman, Yusen, Kline.

Study supervision: Jimenez, Yusen.

The corresponding author, David Jiménez, had full access to all the data in the study and had final responsibility for the decision to submit for publication. 
Diclofenac for intermediate-risk PE

Thromb Res

\section{REFERENCES}

1. Smulders Y. Pathophysiology and treatment of haemodynamic instability in acute pulmonary embolism: the pivotal role of pulmonary vasoconstriction. Cardiovasc Res 2000; 48: 23-33.

2. Nordenholz KE, Mitchell AM, Kline JA. Direct comparison of the diagnostic accuracy of fifty protein biological markers of pulmonary embolism for use in the emergency department. Acad Emerg Med 2008; 15: 795-799.

3. Mitchell AM, Nordenholz KE, Kline JA. Tandem measurement of D-dimer and myeloperoxidase of $\mathrm{C}$-reactive protein to effectively screen for pulmonary embolism in the emergency department. Acad Emerg Med 2008; 15: 800-805.

4. Kearon C, Kahn SR, Agnelli G, Goldhaber S, Raskob GE, Comerota AJ. Antithrombotic therapy for venous thromboembolic disease: American College of Chest Physicians Evidence-Based Clinical Practice Guidelines (8th Edition). Chest 2008; 133: 454S-545S.

5. Verstraete M, Miller GA, Bounameaux H, Charbonnier B, Colle JP, Lecorf G, Marbet GA, Mombaerts P, Olsson CG. Intravenous and intrapulmonary recombinant tissue-type plasminogen activator in the treatment of acute massive pulmonary embolism. Circulation 1988; 77: 353-60.

6. Chatterjee S, Chakraborty A, Weinberg I, Kadakia M, Wilensky RL, Sardar P, Kumbhani DJ, Mukherjee D, Jaff MR, Giri J. Thrombolysis for pulmonary embolism and risk of all-cause mortality, major bleeding, and intracraneal hemorrhage. JAMA 2014; 311: 2414-2421.

7. Marti C, John G, Konstantinides S, Slattery D, Fanikos J, O'Neil BJ, Thompson JR, Hiestand B, Briese BA, Pendleton RC, Miller CD, Kline JA. Systemic thrombolytic therapy for acute pulmonary embolism: a systematic review and metaanalysis. Eur Heart J 2015; 36: 605-614.

8. Pollack CV, Schreiber D, Goldhaber SZ, et al. Clinical characteristics, management, and outcomes of patients diagnosed with acute pulmonary embolism in the emergency department Initial Report of EMPEROR (Multicenter Emergency Medicine Pulmonary Embolism in the Real World Registry). J Am Coll Cardiol 2011; 57: 700-6. 
9. Watts JA, Gellar MA, Stuart LK, Obraztsova M, Kline JA. Proinflammatory events in right ventricular damage during pulmonary embolism: effects of treatment with ketorolac in rats. J Cardiovasc Pharmacol 2009; 54: 246-252.

10. PIOPED investigators. Value of ventilation/perfusion scan in acute pulmonary embolism: results of the prospective investigation of the pulmonary embolism diagnosis (PIOPED). JAMA 1990; 263: 2753-2759.

11. Remy-Jardin M, Remy J, Wattinne L, Giraud F. Central pulmonary thromboembolism: diagnosis with spiral volumetric CT with the single-breathhold-technique-comparison with pulmonary angiography. Radiology 1992; 185: 381-387.

12. Becattini C, Agnelli G, Vedovati MC, Pruszczyk P, Casazza F, Grifoni S, Salvi A, Bianchi M, Douma R, Konstantinides S, Lankeit M, Duranti M. Multidetector computed tomography for acute pulmonary embolism: diagnosis and risk stratification in a single test. Eur Heart J 2011; 32: 1657-1663.

13. Schulman S, Kearon C. Definition of major bleeding in clinical investigations of antihemostatic medicinal products in non-surgical patients. J Thromb Haemost 2005; 3: 692-694.

14. Becattini C, Agnelli G, Salvi A, Grifoni S, Pancaldi LG, Enea I, Balsemin F, Campanini M, Ghirarduzzi A, Casazza F; TIPES Study Group. Bolus tenecteplase for right ventricular dysfunction in hemodynamically stable patients with pulmonary embolism. Thromb Res 2010; 125: e82-e86.

15. Altman DG, Bland JM. Measurement in medicine: the analysis of method comparison studies. Statistician. 1983; 32: 307-317.

16. Sohn HY, Krotz F. Cyclooxygenase inhibition and atherothrombosis. Curr Drug Targets 2006; 7: 1275-1284.

17. Gislason GH, Rasmussen JN, Abildstrom SZ, Schramm TK, Hansen ML, Fosbøl EL, Sørensen R, Folke F, Buch P, Gadsbøll N, Rasmussen S, Poulsen HE, Køber L, Madsen M, Torp-Pedersen C. Increased mortality and cardiovascular morbidity associated with use of nonsteroidal antiinflammatory drugs in chronic heart failure. Arch Intern Med 2009; 169: 141-149. 
18. Ray WA, Varas-Lorenso C, Chung CP, Castellsague J, Murray KT, Stein CM, Daugherty JR, Arbogast PG, García-Rodríguez LA. Cardiovascular risks of nonsteroidal antiinflammatory drugs in patients after hospitalization for serious coronary heart disease. Circ Cardiovasc Qual Outcomes 2009; 2: 155-163.

19. Gan TJ. Diclofenac: an update on its mechanism of action and safety profile. Curr Med Res Opin 2010; 26: 1715-1731.

20. Campbell WI, Kendrick R, Patterson C. Intravenous diclofenac sodium. Does its administration before operation suppress postoperative pain? Anaesthesia 1990; 45: 763-766.

21. Rorarius MG, Baer GA, Siirtola M, Lahti T, Laippala P. Effect of intravenous diclofenac or indomethacin on the emergence from anaesthesia for tonsillectomy. Acta Anaesthesiol Scand 1993; 37: 616-621.

22. Davidson BL, Verheijen S, Lensing AW, Gebel M, Brighton TA, Lyons RM, Rehm J, Prins MH. Bleeding risk of patients with acute venous thromboembolism taking nonsteroidal anti-inflammatory drugs or aspirin. JAMA Intern Med 2014; 174: 947-953.

23. Sanchez O, Trinquart L, Colombet I, Durieux P, Huisman MV, Chatellier G, Meyer G. Prognostic value of right ventricular dysfunction in patients with haemodynamically stable pulmonary embolism: a systematic review. Eur Heart J 2008; 29: 1569-77.

24. Kucher N, Boekstegers P, Müller OJ, Kupatt C, Beyer-Westendorf J, Heitzer T, Tebbe U, Horstkotte J, Müller R, Blessing E, Greif M, Lange P, Hoffmann RT, Werth S, Barmeyer A, Härtel D, Grünwald H, Empen K, Baumgartner I. Randomized, controlled trial of ultrasound-assisted catheter-directed thrombolysis for acute intermediate-risk pulmonary embolism. Circulation 2014; 129: 479-486.

25. Dalen JE, Alpert JS, Hirsch J. Thrombolytic therapy for pulmonary embolism: is it effective? Is it safe? When is it indicated? Arch Intern Med 1997; 157: 2550-2556. 
Diclofenac for intermediate-risk PE

Thromb Res

26. Rudski LG, Lai WW, Afilalo J, Hua L, Handschumacher MD, Chandrasekaran K, Solomon SD, Louie EK, Schiller NB. Guidelines for the echocardiographic assessment of the right heart in adults: a report from the American Society of Echocardiography endorsed by the European Association of Echocardiography, a registered branch of the European Society of Cardiology, and the Canadian Society of Echocardiography. J Am Soc Echocardiogr 2010; 23: 685-713.

27. Jiménez D, Kopecna D, Tapson V, Briese B, Schreiber D, Lobo JL, Monreal M, Aujesky D, Sanchez O, Meyer G, Konstantinides S, Yusen RD, On Behalf Of The Protect Investigators. Derivation and validation of multimarker prognostication for normotensive patients with acute symptomatic pulmonary embolism. Am J Respir Crit Care Med 2014; 189: 718-726. 
Diclofenac for intermediate-risk PE Thromb Res

Figure 1. Patient Flow Diagram

Figure 2. RV/LV ratio at baseline and at 48 hours for each patient in (A) the diclofenac group and in (B) the placebo group.

Figure 3. Baseline RV/LV ratio: investigator vs. core laboratory 
Diclofenac for intermediate-risk PE Thromb Res

\section{Figure 1.}

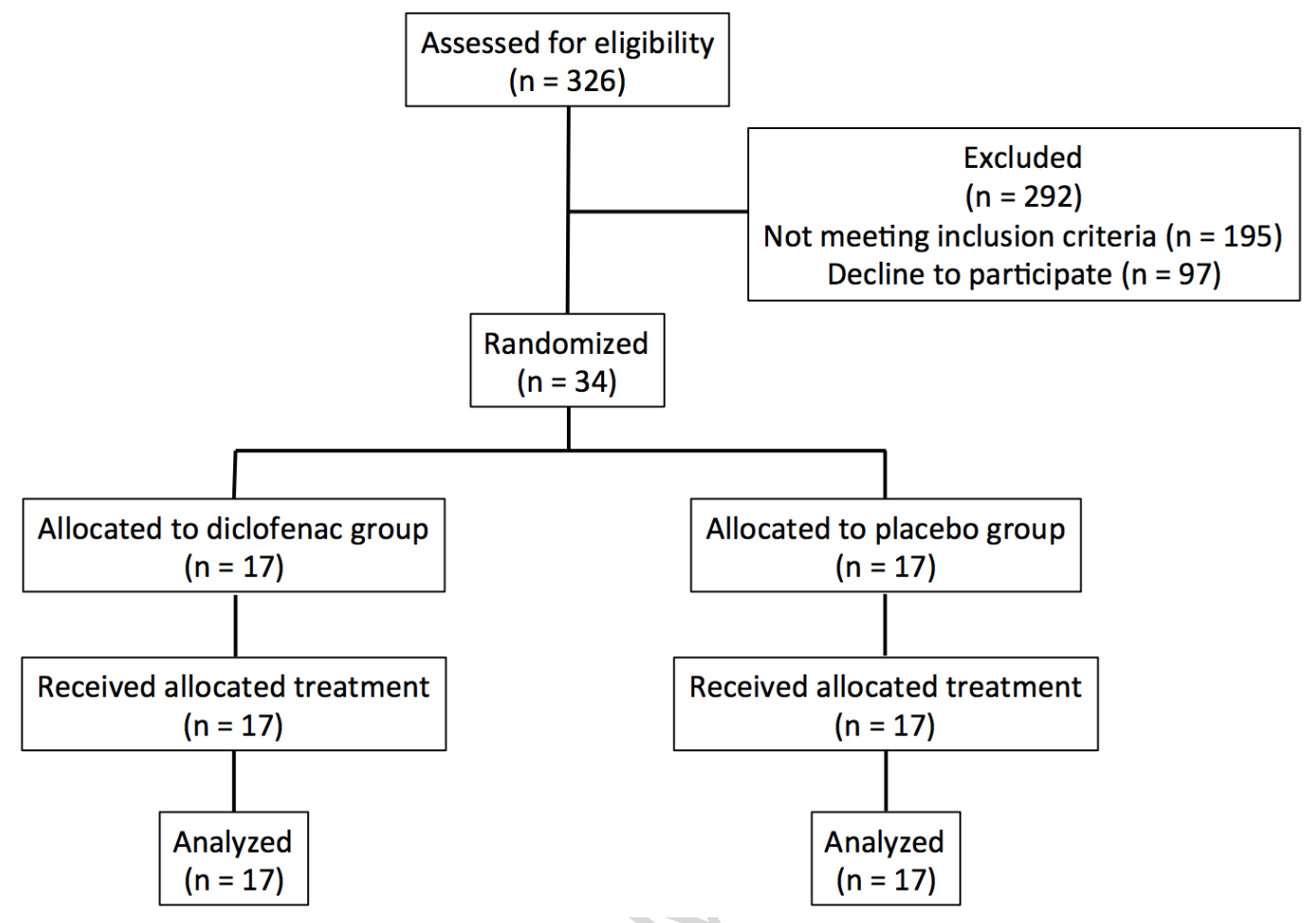


Diclofenac for intermediate-risk PE Thromb Res

Figure 2.

Diclofenac

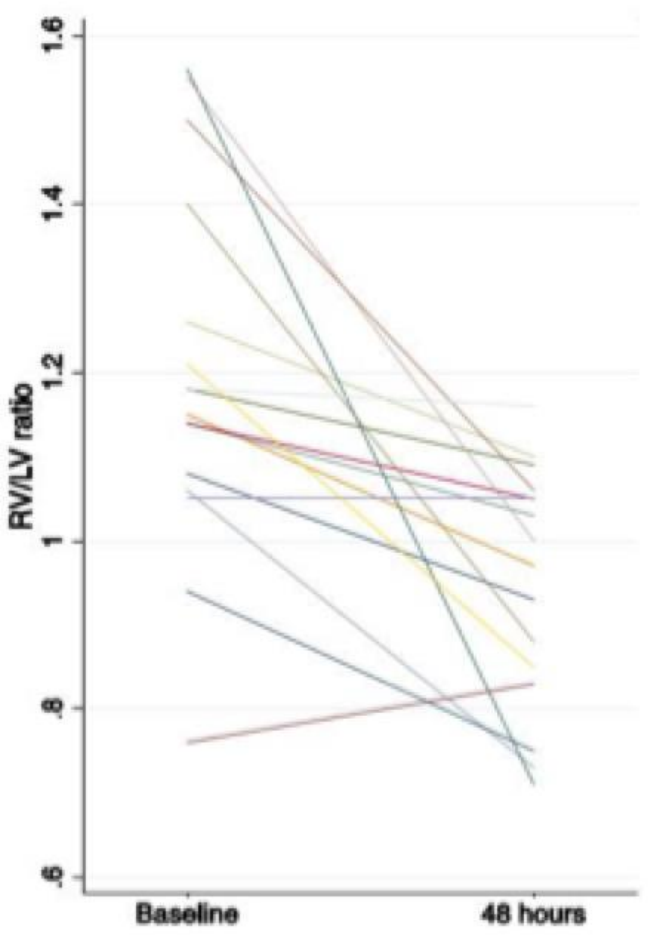

$1.2 \pm 0.2 \mathrm{~mm}$

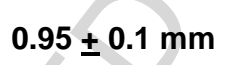

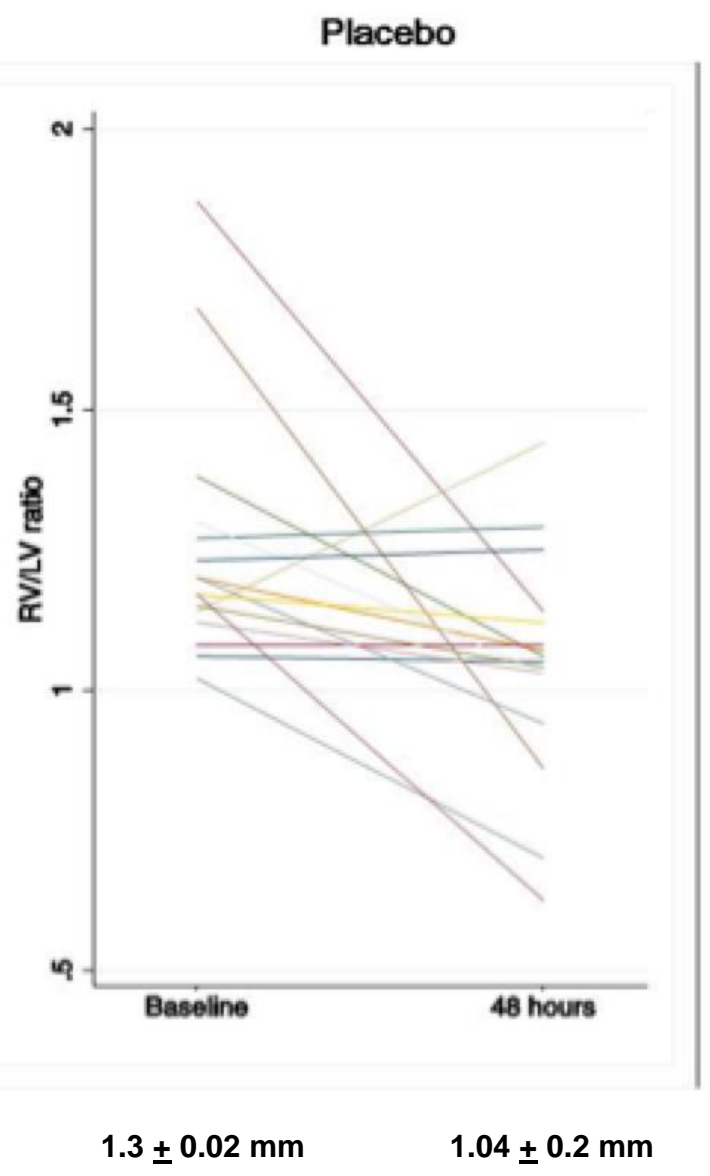

Abbreviations: RV, right ventricle; $L V$, left ventricle. 
Diclofenac for intermediate-risk PE Thromb Res

Figure 3.

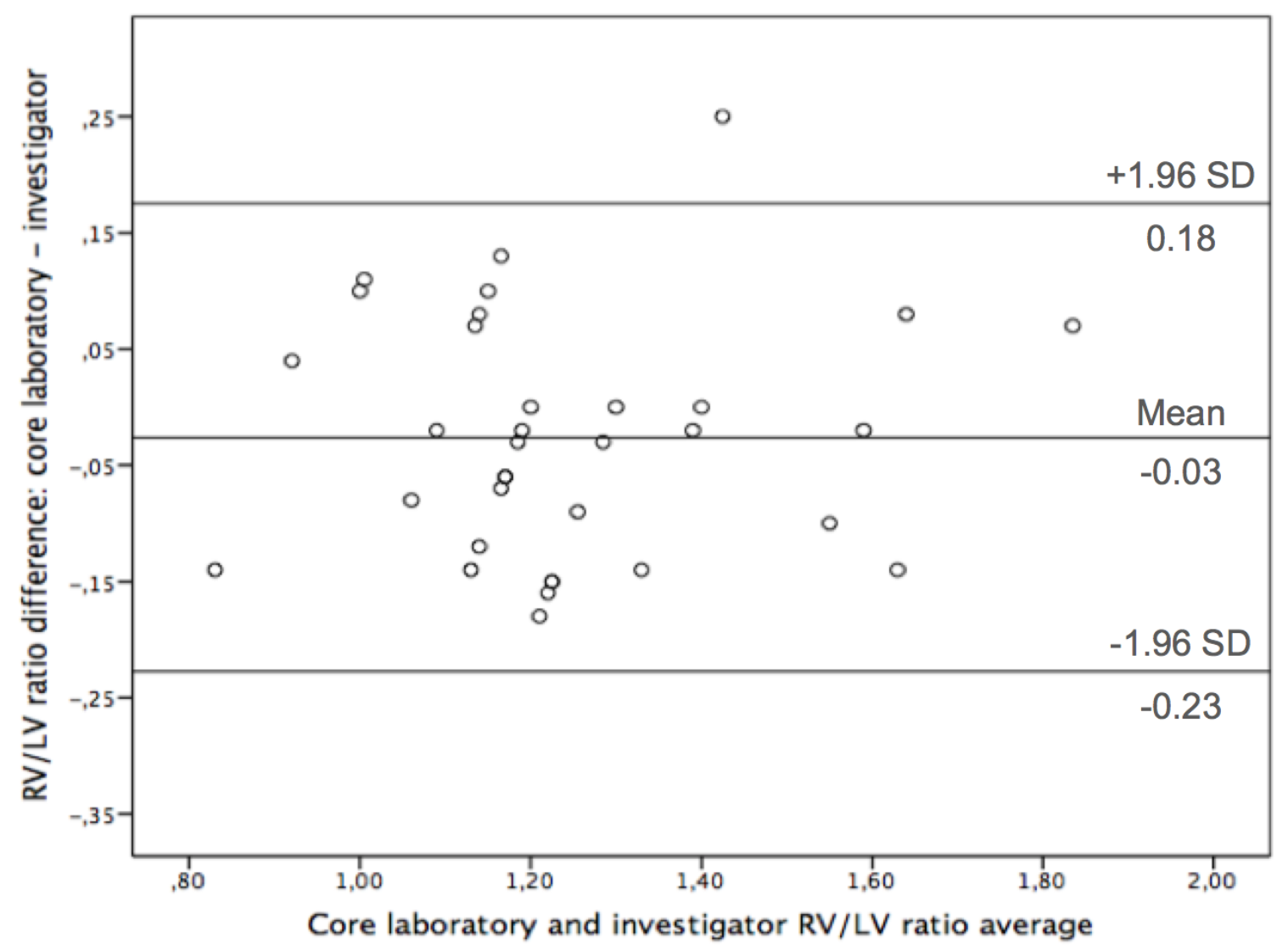

Abbreviations: RV, right ventricle; LV, left ventricle; SD, standard deviation. 
Diclofenac for intermediate-risk PE

Thromb Res

\section{Table 1. Characteristics of the patients at baseline ${ }^{\star}$}

\begin{tabular}{lcc}
\hline Characteristic & Diclofenac & Placebo \\
& $(\mathrm{N}=17)$ & $(\mathrm{N}=17)$
\end{tabular}

\begin{tabular}{lcc}
\hline Demographic data & & \\
Age, years & $73.7 \pm 10.3$ & $67.5 \pm 17.2$ \\
$\quad$ Mean & $76.0(69.0-78.0)$ & $69.0(58.5-80.0)$ \\
$\quad$ Median (interquartile range) & $7(41.2)$ & $5(29.4)$ \\
Male sex, $\mathrm{n}(\%)$ & $79.9 \pm 13.2$ & $73.4 \pm 20.2$ \\
Mean weight, Kg & & \\
Clinical status & $132.3 \pm 12.6$ & $126.0 \pm 18.9$ \\
Systolic blood pressure, mm Hg & $98.5 \pm 26.5$ & $101.7 \pm 16.0$ \\
Heart rate, beats per minute & $87.7 \pm 5.2$ & $87.6 \pm 8.6$ \\
Arterial oxyhemoglobin saturation, \% & & \\
Medical history & $0(0)$ & $0(0)$ \\
Chronic pulmonary disease, $\mathrm{n}(\%)$ & $0(0)$ & $0(0)$ \\
Chronic heart failure, $\mathrm{n}(\%)$ & $1(5.9)$ & $2(11.8)$ \\
Previous venous thromboembolism, $\mathrm{n}(\%)$ & $0(0)$ & $2(11.8)$ \\
Active cancer, $\mathrm{n}(\%)$ & $2(11.8)$ & $1(5.9)$ \\
Surgery or major trauma in the previous month, $\mathrm{n}(\%)$ & $3(17.6)$ & $4(23.5)$ \\
Immobilization, $\mathrm{n}(\%)$ & & \\
Simplified PESI & $5(29 \%)$ & $4(24 \%)$ \\
Low-risk & $12(71 \%)$ & $13(76 \%)$ \\
High-risk & & \\
\hline
\end{tabular}

Abbreviations: PESI, Pulmonary Embolism Severity Index.

* Plus-minus values are means \pm SD. Between-group differences in the characteristics listed here were not significant. 
Diclofenac for intermediate-risk PE

Thromb Res

Table 2. Echocardiographic laboratory data

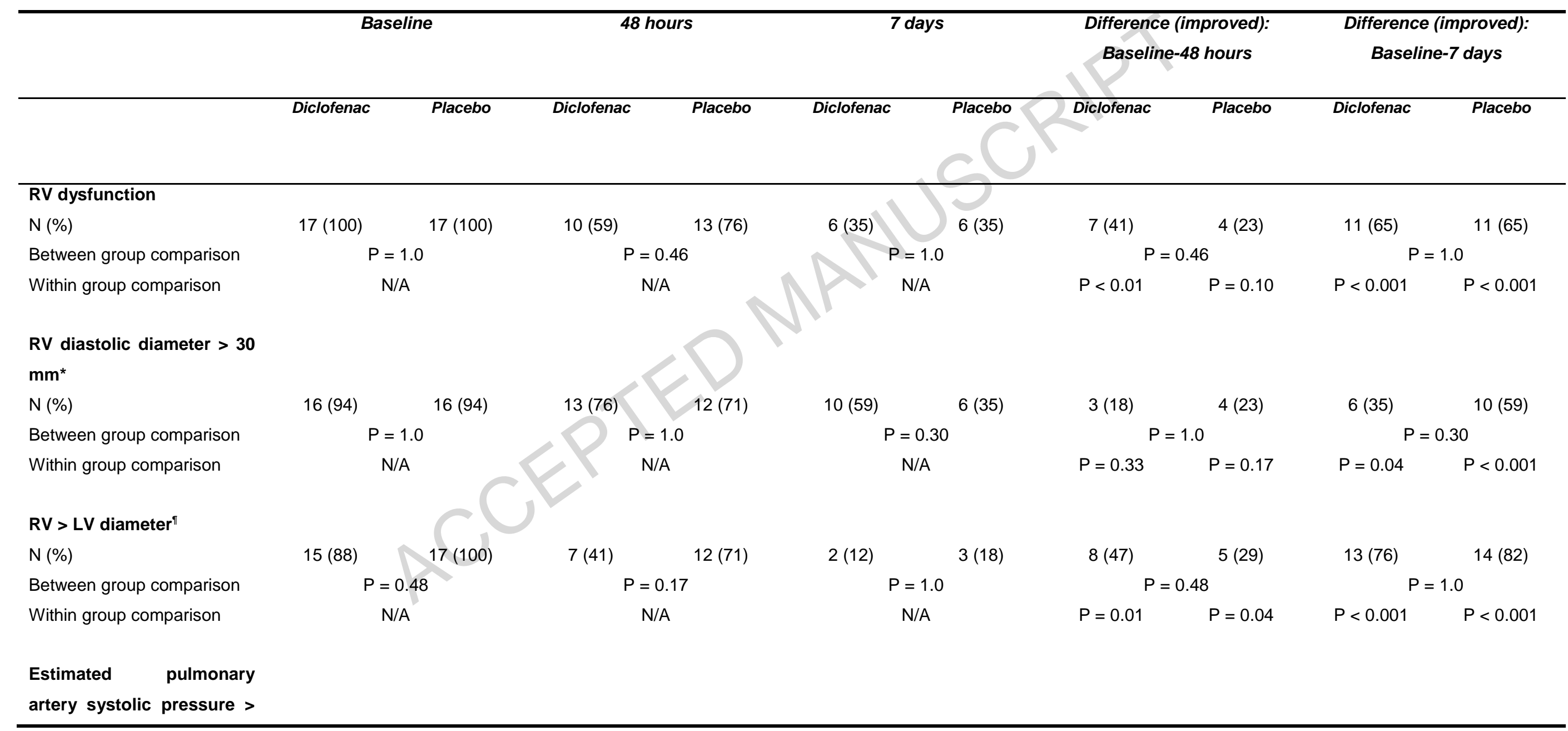


Diclofenac for intermediate-risk PE

Thromb Res

\begin{tabular}{|c|c|c|c|c|c|c|c|c|c|c|}
\hline \multicolumn{11}{|l|}{$30 \mathrm{~mm} \mathrm{Hg}$} \\
\hline N (\%) & $15(88)$ & $17(100)$ & $9(53)$ & $10(59)$ & $6(35)$ & $7(41)$ & 6 (35) & $7(41)$ & $9(53)$ & $10(59)$ \\
\hline Between group comparison & \multicolumn{2}{|c|}{$P=0.48$} & \multicolumn{2}{|c|}{$P=1.0$} & \multicolumn{2}{|c|}{$P=1.0$} & \multicolumn{2}{|c|}{$P=1.0$} & \multicolumn{2}{|c|}{$P=1.0$} \\
\hline Within group comparison & \multicolumn{2}{|c|}{$\mathrm{N} / \mathrm{A}$} & \multicolumn{2}{|c|}{$\mathrm{N} / \mathrm{A}$} & \multicolumn{2}{|c|}{$N / A$} & & $P<0.01$ & $P<0.01$ & $P<0.001$ \\
\hline \multicolumn{11}{|l|}{$\begin{array}{l}\text { Hypokinesis of the RV free } \\
\text { wall }\end{array}$} \\
\hline$N(\%)$ & $13(76)$ & $12(71)$ & $7(41)$ & $7(41)$ & $2(12)$ & & $6(35)$ & $5(29)$ & $11(65)$ & $11(65)$ \\
\hline Between group comparison & \multicolumn{2}{|c|}{$P=1.0$} & \multicolumn{2}{|c|}{$P=1.0$} & & & \multicolumn{2}{|c|}{$P=1.0$} & \multicolumn{2}{|c|}{$P=1.0$} \\
\hline Within group comparison & \multicolumn{2}{|c|}{$\mathrm{N} / \mathrm{A}$} & \multicolumn{2}{|c|}{$N / A$} & & & $P=0.08$ & $P=0.17$ & $P<0.001$ & $P<0.001$ \\
\hline \multicolumn{11}{|l|}{ TAPSE, mm } \\
\hline Mean \pm SD & $17.7 \pm 4.1$ & $16.8 \pm 4.4$ & $19.8 \pm 3.8$ & $18.4 \pm 3.4$ & $20.9 \pm 3.8$ & $19.7 \pm 3.3$ & $2.6 \pm 3.1$ & $1.3 \pm 1.6$ & $3.2 \pm 3.6$ & $4.1 \pm 3.7$ \\
\hline Between group comparison & \multicolumn{2}{|c|}{$P=0.53$} & \multirow{2}{*}{\multicolumn{2}{|c|}{$P=0.30$}} & \multicolumn{2}{|c|}{$P=0.39$} & \multicolumn{2}{|c|}{$P=0.14$} & \multicolumn{2}{|c|}{$P=0.55$} \\
\hline Within group comparison & \multicolumn{2}{|c|}{$\mathrm{N} / \mathrm{A}$} & & & \multicolumn{2}{|c|}{$\mathrm{N} / \mathrm{A}$} & $P<0.001$ & $P<0.001$ & $P<0.001$ & $P<0.001$ \\
\hline
\end{tabular}

Abbreviations: RV, right ventricle; N/A, not applicable; LV, left ventricle; TAPSE, tricuspid annular pulmonary systolic excursion; SD, standard deviation.

${ }^{*}$ Measured in the parasternal window.

"In the apical or subcostal space. 
Diclofenac for intermediate-risk PE

Thromb Res

\section{Table 3. Clinical events at 30-days}

\begin{tabular}{lcc}
\hline Clinical event & $\begin{array}{c}\text { Diclofenac } \\
(\boldsymbol{n}=\mathbf{1 7})\end{array}$ & $\begin{array}{c}\text { Placebo } \\
(\boldsymbol{n}=\mathbf{1 7})\end{array}$ \\
\hline All-cause mortality & 0 & 0 \\
PE-related mortality & 0 & 0 \\
Haemodynamic collapse $^{*}$ & 0 & $1^{\pi}$ \\
Recurrent VTE & 0 & $1^{\pi}$ \\
Major bleeding & 0 & $1^{\pi}$ \\
\hline
\end{tabular}

Abbreviations: PE, pulmonary embolism; VTE, venous thromboembolism.

* Haemodynamic collapse was defined as a systolic blood pressure $<90 \mathrm{~mm} \mathrm{Hg}$ for at least 15 minutes, need for catecholamine administration because of persistent arterial hypotension or shock, need for thrombolysis, need for endotracheal intubation, or need for cardiopulmonary resuscitation.

" All events occurred in the same patient. 
Diclofenac for intermediate-risk PE

Thromb Res

\section{HIGHLIGHTS}

- Anti-inflammatory drugs may facilitate the reversal of RV dysfunction in PE.

- We assessed the efficacy of diclofenac for reversing RV dysfunction in PE.

- Our study is inconclusive as to a potential benefit of diclofenac in PE. 